\title{
Interaction Effects in Conductivity of a Two-Valley Electron System in High-Mobility Si Inversion Layers
}

\author{
N. N. Klimovi,3, D. A. Knyazev², O. E. Omel'yanovskii ${ }^{2}$, \\ V. M. Pudalov ${ }^{2}$, H. Kojima ${ }^{1}$, and M. E. Gershenson ${ }^{1}$ \\ ${ }^{1}$ Department of Physics and Astronomy, \\ Rutgers University, \\ New Jersey 08854, USA. \\ ${ }^{2}$ P. N. Lebedev Physical Institute, \\ Moscow 119991, Russia. \\ 3 P. N. Lebedev Physics Research Center, \\ Moscow 119991, Russia.
}

(Dated: October 25, 2018)

\begin{abstract}
We have measured the conductivity of high-mobility (001) Si metal-oxide-semiconductor field effect transistors (MOSFETs) over wide ranges of electron densities $n=(1.8-15) \times 10^{11} \mathrm{~cm}^{-2}$, temperatures $T=30 \mathrm{mK}-4.2 \mathrm{~K}$, and in-plane magnetic fields $B_{\|}=(0-5) \mathrm{T}$. The experimental data have been analyzed using the theory of interaction effects in the conductivity $\sigma$ of disordered $2 \mathrm{D}$ systems. The parameters essential for comparison with the theory, such as the intervalley scattering time and valley splitting, have been measured or evaluated in independent experiments. The observed behavior of $\sigma$, including its quasi-linear increase with decreasing $T$ down to $\sim 0.4 \mathrm{~K}$ and its downturn at lower temperatures, is in agreement with the theory. The values of the Fermiliquid parameter obtained from the comparison agree with the corresponding values extracted from the analysis of Shubnikov-de Haas oscillations based on the theory of magnetooscillations in interacting $2 \mathrm{D}$ systems.
\end{abstract}

PACS numbers: PACS: 


\section{INTRODUCTION}

The beginning of the 80's witnessed a triumph of the one-parameter scaling theory of localization ${ }^{1}, 2$ and the theory of electron-electron interactions in disordered conductors (for a review, see Ref. 3). As a result, the peculiar low-temperature behavior of the conductivity of numerous low-dimensional systems has been successfully attributed to quantum interference effects (see, e.g., Ref. 4). Curiously, application of these ideas to the two-dimensional (2D) electron liquid in Si MOSFETs - one of the most ubiquitous 2D systems - remained a challenge for more than 25 years. In the early experiments ${ }^{5} \underline{\underline{6}}$ with low-mobility $\left(\mu \sim 0.1 \mathrm{~m}^{2} / \mathrm{Vs}\right)$ structures in the regime of relatively high electron densities $\left(n>10^{12} \mathrm{~cm}^{-2}\right)$, a decrease of the conductivity with cooling has been observed, apparently in qualitative agreement with the ideas of weak localization $\frac{1,7}{n}$ and electron-electron interactions ${ }^{3}$. However, the quantitative description of this behavior remained a problem ${ }^{8}$. The disagreement with the theory became qualitative with the advent of high-mobility $\left(\mu \geq 2 \mathrm{~m}^{2} / \mathrm{Vs}\right)$ structures: the low $-T$ conductivity of high- $\mu$ Si MOSFETs increased with decreasing temperature ${ }^{9}$, in striking contrast to the behavior of many other $2 \mathrm{D}$ systems. This "metallic" behavior of the conductivity is especially pronounced at low electron densities $n \sim(1-2) \times 10^{11} \mathrm{~cm}^{-2}$, where a five-fold increase of $\sigma(T)$ with cooling was observed $\underline{10}, \underline{11}, \underline{12}, \underline{13}$. Later, the quasi-linear increase of $\sigma(T)$ was observed in many high-mobility 2D systems in the "dilute" regime, including GaAs heterostructures (both $p$-type $\frac{14,15,16,17}{17}$ and $n$-type $\left.\frac{18,19}{19}\right), \mathrm{Si}_{\mathrm{SiGe}} \mathrm{Si}^{20}$, and $\mathrm{AlAs}^{21}$ quantum wells.

The subsequent development of theory and experiment led to significant progress in our understanding of the low-temperature transport in high-mobility systems in the regime of low electron densities. In the mid-80's, the quasi-linear "metallic" dependence observed in the ballistic interaction regime $T \tau \gg 1$ ( $\tau$ is the transport mean free time, here and below we set $\hbar=k_{B}=e=1$ ) was attributed ${ }^{22}$ to weakening of the screening of the scattering potential with increasing $T$. Observation of a strong negative magnetoconductance induced by the in-plane magnetic field 23 and renormalization of the effective electron mass and $g$-factor in these structures 24,25 also suggested that the electron-electron interactions play an important role in this phenomenon. More recently, Zala, Narozhny, and Aleiner (ZNA) ${ }^{26}$ developed a theory that took into account all interaction contributions to the conductivity, including the exchange ones. This theory offers a unified approach to both ballistic $(T \tau \gg 1)$ and diffusive 
$(T \tau \ll 1)$ interaction regimes by considering the quantum interference between electron waves scattered off a short-range random potential "dressed" by Friedel oscillations of the electron density. The theory was extended for the case of a long-range scattering potential by Gornyi and Mirlin (GM) $\underline{27}$. The theories $\underline{26}, 27$ naturally incorporate the Altshuler-Aronov results for the interaction corrections to the conductivity in the diffusive regime ${ }^{3}$.

For the diffusive regime, a more general approach to interacting systems based on the nonlinear $\sigma$-model has been developed by Finkel'stein 28 . Recently, the renormalization group $(\mathrm{RG})$ equations of this theory 29,30 (obtained in the first order in $\frac{1}{\pi \sigma}$ and in all orders in interaction) have been compared with the conductivity of Si MOSFETs at low electron densities $29,31,32,33$.

The RG equations $28,29,30,34,35$ describe the length scale (temperature) evolution of the resistivity and interaction parameters for a $2 \mathrm{D}$ electron system in the diffusive regime ${ }^{28}$. However, at high electron densities, the temperature range corresponding to the diffusive regime shrinks. In contrast, the theory of interaction corrections 26,27 is applicable over a wider $T$ range (that includes both ballistic and diffusive regimes) provided $\sigma \gg 1$ and $\Delta \sigma / \sigma \ll 1 ;$ these assumptions are well justified at high densities.

The theories 26,27 predict that the magnitude and sign of the the interaction correction $\Delta \sigma(T, B)$ is determined by the value of the Fermi-liquid parameter $F_{0}^{\sigma}$ (which can be found by measuring the Shubnikov-de Haas $(\mathrm{SdH})$ oscillations in weak magnetic fields perpendicular to the plane of a $2 \mathrm{D}$ structure $\frac{24,25}{2}$ or the magnetoresistance in strong in-plane magnetic fields $\left.s^{36}\right)$. In particular, it is expected that the $\sigma(T)$ dependence becomes "metallic" when $F_{0}^{\sigma}$ is negative and its absolute value exceeds a certain threshold (see Sec. II).

The experimental studies of the conductivity in various low-carrier-density 2D systems in the ballistic (high-temperature) regime are in agreement with the ZNA and GM theories. The "metallicity" in all these systems is enhanced at low $n$ due to an increase of the absolute value of $F_{0}^{\sigma}$ (see, e.g., Refs. 37,38,39). In Si MOSFETs, the interaction effects are especially strong due to the presence of two nearly degenerate valleys in the electron spectrum 29 . This enhancement, however, diminishes if the temperature $T$ becomes smaller than the valley splitting $\Delta_{V}$ and intervalley scattering rate $\tau_{v}^{-1}$. As a result, with lowering $T$, the "metallic" dependence of $\sigma(T)$ is expected to become weaker or even to be replaced with an "insulating" one. To the best of our knowledge, this behavior has not been observed for Si MOSFETs prior to our work. 
This paper aims to study in detail the conductivity of 2D electron liquid in high-mobility (001) Si MOSFETs over a wide temperature range $(T=0.03-4.2 \mathrm{~K})$ that includes both the diffusive and ballistic regimes. In particular, we observed for the first time that the "metallic" increase of $\sigma$ with cooling is followed by the downturn of $\sigma(T)$ at lower temperatures. For the purpose of comparison with the ZNA theory ${ }^{26}$, we studied the range of not-too-low densities, $n=(1.8-15) \times 10^{11} \mathrm{~cm}^{2}$, where the temperature and magnetic field dependences $\Delta \sigma(T, B)$ can still be treated as small corrections to the Drude conductivity $\sigma_{D}$. In principle, no fitting parameters are required for comparison with the theory, because we have measured $F_{0}^{\sigma}, \Delta_{V}$, and $\tau_{v}$ in independent experiments. However, below we take a slightly different approach: we obtain the $F_{0}^{\sigma}(n)$ values from fitting the $\Delta \sigma\left(T, B_{\|}\right)$dependences with the ZNA theory ${ }^{26}$, and show that these values are consistent with the corresponding values extracted from the analysis of $\mathrm{SdH}$ oscillations 25 . We have also revealed shortcomings of earlier analysis of $\Delta \sigma(T)$, reanalyzed the available data, and compared the extracted values of $F_{0}^{\sigma}(n)$ with corresponding values from other measurements. We conclude that the experimental data are well described by the theory of interaction corrections 26 at intermediate temperatures $T \approx(0.3-4.2) \mathrm{K}$. For a quantitative analysis at ultra-low temperatures $(T \leq 0.3 \mathrm{~K})$, the interaction correction theory should be modified by taking into account finite intervalley scattering rates.

The paper is organized as follows. In Section II we briefly summarize the theoretical results $^{26}$ for the interaction corrections to the conductivity of a two-valley system. The experimental data are presented in Section III, along with the data analysis and discussion. The summary is given in Section IV.

\section{INTERACTION CORRECTIONS TO THE CONDUCTIVITY}

\section{A. Temperature dependence of the conductivity in zero magnetic field}

In the ZNA theory ${ }^{26}$, the corrections $\Delta \sigma_{e e}$ to the Drude conductivity $\sigma_{D}=n \tau / m_{b}\left(m_{b} \approx\right.$ $0.205 m_{e}$ is the electron band mass in (001) Si MOSFETs; for more detail, see discussion in section IIIA3 and references therein) were calculated in both ballistic and diffusive regimes for all orders of the interaction strength and the leading order in $1 /\left(E_{F} \tau\right)$ and $T / E_{F}$. In particular, the theory reproduces the Altshuler-Aronov correction $\underline{3}$ to the conductivity in the 
diffusive regime. To adapt the theoretical results ${ }^{26}$ to the case of (001) Si MOSFETs, one should take into account that the electron spectrum in this system has two almost degenerate valleys $\stackrel{40}{ }$. In zero magnetic field, $\Delta \sigma_{e e}(T)$ for a system with two degenerate valleys in the absence of intervalley scattering can be written as follows 38 :

$$
\Delta \sigma_{e e}(T)=\delta \sigma_{C}(T)+15 \delta \sigma_{T}(T)
$$

Here $\delta \sigma_{C}$ is the so-called "charge" contribution which combines Fock correction and the singlet part of Hartree correction, and $\delta \sigma_{T}$ is the "triplet" contribution due to the triplet part of Hartree term. The valley index can be considered as a pseudo-spin in multi-valley systems $^{29}$, and the valley degeneracy determines the number of triplet terms due to the spin exchange processes between electrons in different valleys. For a system with two degenerate valleys, the total number of interaction channels is $4 \times 4=16$, among them 1 singlet and 15 triplet terms (for comparison, there are 1 singlet and 3 triplet terms for a single-valley system).

Below we assume that the scattering potential is short-ranged which is relevant to $\mathrm{Si}$ MOSFETs. According to Ref. 26, the charge term does not depend on the details of interactions:

$$
\delta \sigma_{C}=\frac{1}{\pi}\left\{(T \tau)\left(1-\frac{3}{8} f(T \tau)\right)-\frac{1}{2 \pi} \ln \frac{E_{F}}{T}\right\},
$$

whereas the magnitude and sign of the triplet term is controlled by the Fermi-liquid $\operatorname{parameter}^{26} F_{0}^{\sigma}$ :

$$
\delta \sigma_{T}=\frac{1}{\pi}\left\{(T \tau) \frac{F_{0}^{\sigma}}{1+F_{0}^{\sigma}}\left(1-\frac{3}{8} t\left(T \tau ; F_{0}^{\sigma}\right)\right)-\left(1-\frac{\ln \left(1+F_{0}^{\sigma}\right)}{F_{0}^{\sigma}}\right) \frac{1}{2 \pi} \ln \frac{E_{F}}{T}\right\} .
$$

The functions $f$ and $t$ in Eqs. (2, 3) describe the crossover between the diffusive $\left(\Delta \sigma_{e e} \propto \ln T\right)$ and ballistic $\left(\Delta \sigma_{e e} \propto T\right)$ regimes; outside the crossover region, they change the value of $\Delta \sigma_{e e}$ by only a few percent. The explicit expressions for these functions can be found in Ref. 26. The diffusive-ballistic crossover is expected over some temperature range near

$$
T^{*}=\frac{1+F_{0}^{\sigma}}{2 \pi \tau}
$$

Equations (213) describe the quantum corrections in a system with the conductance $\sigma \gg$ $1 / 2 \pi$ at temperatures well below the Fermi energy $\left[T \ll\left(1+F_{0}^{\sigma}\right)^{2} E_{F}\right]$.

The sign and the magnitude of $\Delta \sigma_{e e}$ is controlled by the Fermi-liquid parameter $F_{0}^{\sigma}$. For a rough estimate, deeply in the ballistic regime the $\ln T$ terms and the crossover functions 
$t$ and $f$ in Eqs. (2/3) can be omitted. For example, at $T \tau=10$, the functions $f$ and $t$ contribute $\approx 4 \%$ and $\approx 11 \%$, respectively, to the linear-in- $T \tau$ ballistic terms of Eqs. (23). By neglecting these terms, we find that for a single-valley system the linear dependence $\Delta \sigma_{e e}(T)$ in the ballistic regime becomes "metallic" $(d \sigma / d T<0)$ at $3 F_{0}^{\sigma} /\left(1+F_{0}^{\sigma}\right)<-1$ or $F_{0}^{\sigma}<-0.25$, whereas for a system with two degenerate valleys, the "metallic" $\Delta \sigma_{e e}(T)$ dependences are expected for $15 F_{0}^{\sigma} /\left(1+F_{0}^{\sigma}\right)<-1$ or $F_{0}^{\sigma}<-0.06$. Thus, the valley degeneracy extends the range of $F_{0}^{\sigma}(n)$, and hence, the range of carrier densities $n$ where the conduction exhibits the "metallic" behavior.

\section{B. Magnetoconductivity in the in-plane magnetic field}

The in-plane magnetic field, being coupled mostly to electron spins, provides a useful tool for exploring the interaction effects in the low-temperature conductivity of Si MOSFETs 23 . When the Zeeman energy $E_{Z}=g_{b} \mu_{B} B\left(g_{b}=2\right.$ is the bare $g$-factor, $\mu_{B}$ is the Bohr magneton) becomes much greater than $T$, the number of triplet terms that contribute to $\Delta \sigma_{e e}(T)$ is reduced from 15 to 7 . Similar reduction of triplet terms is expected for a valley splitting $\Delta_{V}>T$. These two effects have been accounted by the theory of interaction corrections $26.38 ;$

in the presence of the magnetic field and/or valley splitting the interaction correction to the conductivity can be expressed as follows 38 :

$$
\begin{aligned}
\Delta \sigma_{e e}\left(T, \tau, F_{0}^{\sigma}, B_{\|}, \Delta_{V}\right) & =\Delta \sigma_{e e}(T)+2 \Delta \sigma^{Z}\left(E_{Z}, T\right) \\
& +2 \Delta \sigma^{Z}\left(\Delta_{V}, T\right)+\Delta \sigma^{Z}\left(E_{Z}+\Delta_{V}, T\right)+\Delta \sigma^{Z}\left(E_{Z}-\Delta_{V}, T\right)
\end{aligned}
$$

where $\Delta \sigma_{e e}(T)$ is given by Eq. (11). All the terms $\Delta \sigma^{Z}(Z, T)$ have a form

$$
\begin{aligned}
& \Delta \sigma^{Z}(Z, T) \equiv \sigma(Z, T)-\sigma(0, T)=\delta \sigma_{b}(Z)+\delta \sigma_{d}(Z)= \\
& \frac{1}{\pi}\left\{\left[\frac{2 F_{0}^{\sigma}}{1+F_{0}^{\sigma}}(T \tau) K_{b}\left(\frac{Z}{2 T}, F_{0}^{\sigma}\right)\right]+\left[K_{d}\left(\frac{Z}{2 \pi T}, F_{0}^{\sigma}\right)\right]+m\left(Z \tau, T \tau ; F_{0}^{\sigma}\right)\right\},
\end{aligned}
$$

if the relevant energies $Z \ll E_{F}$ ( $Z$ stands for $E_{Z}, \Delta_{V}$, and combinations $\left.E_{Z} \pm \Delta_{V}\right)$. The explicit expressions for the functions $K_{b}$ and $K_{d}$ are given in Ref. 26. In particular, Eq. (66) describes the interaction-driven magnetoconductivity in the magnetic fields which are much weaker than the field of full spin polarization of a system. Below we will neglect the function 
$m\left(0, T \tau ; F_{0}^{\sigma}\right)$ which describes the crossover between the ballistic and diffusive regimes: this function is numerically small and does not modify the value of $\Delta \sigma(Z, T)$ outside the ballisticdiffusive crossover region by more then one percent.

It is worth mentioning that in the framework of the RG theory, the magnetoconductance can also be described by the Castellani-Di Castro-Lee formula $\underline{35}, \underline{4}$ which is equivalent to Eq. (5I) in the diffusive limit at $\Delta_{V}=0$. However, for the analysis of our magnetoconductivity data measured over a wide $T$-range that includes both diffusive and ballistic regimes, the interaction correction theory ${ }^{26}$ is more appropriate than the RG theory ${ }^{35}$.

The interaction correction theory $\underline{26}$ (as well as the RG theory $\underline{29} \underline{\underline{30}}$ ) does not take into account intervalley scattering. This approximation is valid when the intervalley scattering rate $\tau_{v}^{-1}$ is much smaller than $T$. In the low-temperature limit $T \ll \tau_{v}^{-1}$, the electron states in different valleys are completely intermixed at the time scale $\sim T^{-1}$ and the correction $\Delta \sigma_{e e}(T)$ for a two-valley system is expected to coincide with that for a single-valley system.

Since the interaction corrections to the conductivity $\Delta \sigma_{e e}(T, B)$ depend on several parameters such as $\tau, F_{0}^{\sigma}, \Delta_{V}$ and $\tau_{v}$, for testing the theoretical results, it is crucial to determine these parameters in independent measurements. This program is realized in the following section.

\section{SAMPLE CHARACTERIZATION AND DATA ANALYSIS}

\section{A. Sample Characterization}

We have studied the temperature and magnetic field dependences of the conductivity for high-mobility (001) Si MOSFETs, which demonstrate the "metallic" quasi-linear $\sigma(T)$ dependences at intermediate temperatures over a wide range of electron densities $n$. In this paper we present the data for two (001) Si MOSFETs, Si6-14 and Si1-46, with the gate oxide thickness $190 \pm 20 \mathrm{~nm}$ and peak mobility $\mu(0.1 \mathrm{~K}) \approx 2 \mathrm{~m}^{2} / \mathrm{Vs}$. The $\sigma(T, B)$ dependences were measured over the temperature range $T=0.03-4.2 \mathrm{~K}$ using a standard low-frequency fourterminal technique. The measuring current was chosen sufficiently small $(I \sim 1-3 \mathrm{nA})$ to avoid overheating of electrons within this temperature range $\frac{42}{2}$. Our experimental setup allowed us to independently control the magnetic field normal to the plane of 2D layer $\left(B_{\perp} \approx-1.5 \ldots+1.5 \mathrm{~T}\right)$ and the in-plane magnetic field $\left(B_{\|} \approx-8 \ldots+8 \mathrm{~T}\right) ;$ this cross-magnetic 
field technique has been described in Ref. 43. Unless otherwise stated, an in-plane magnetic field of $B_{\|}=0.02 \mathrm{~T}$ was applied to quench the superconductivity in the current/voltage contact pads and the gate electrode which are made of thin aluminum films.

1. Effective mass $m^{*}$ and $g$-factor. Interaction corrections to the magnetooscillations.

The electron density $n$, the electron temperature $T$, the effective electron mass $m^{*}$, and $g$-factor $g^{*}$ have been found from measurements of SdH oscillations (see also Ref.25). For fitting the oscillations, as the first step of the analysis, we have used Lifshitz-Kosevich (LK) formula $\frac{44}{}$ which is valid for non-interacting $2 \mathrm{D}$ electrons if the amplitude of oscillations is small 15 :

$$
\begin{aligned}
& \frac{\delta \sigma_{x x}\left(\omega_{c}, T\right)}{\sigma_{D}}=\sum_{s} A_{s}^{\mathrm{LK}}\left(\omega_{c}, T\right) \cos \left[\pi s\left(\frac{2 E_{F}}{\omega_{c}}-1\right)\right] \cos \left[\pi s \frac{g^{*} m^{*}}{2 m_{e}}\right] \cos \left[\pi s \frac{\Delta_{V}}{\omega_{c}}\right] . \\
& A_{s}^{\mathrm{LK}}\left(\omega_{c}, T\right)=-4 \exp \left(-\frac{2 \pi^{2} s T_{D}}{\omega_{c}}\right) \frac{2 \pi^{2} s T / \omega_{c}}{\sinh \left(2 \pi^{2} s T / \omega_{c}\right)}
\end{aligned}
$$

Here $\omega_{c}=B_{\perp} / m^{*}$ is the cyclotron frequency, $T_{D}=1 / 2 \pi \tau_{D}$ is the Dingle temperature, $\tau_{D}$ is the elastic quantum scattering time. Figure 1 shows that the temperature dependence of the amplitude of the first harmonic, $A_{1}(T)$, is in agreement with Eq. (77) down to the lowest temperatures; this indicates that the electrons are not overheated (with respect to the thermal bath) by the bias current and/or noise.

The $F_{0}^{\sigma}\left(r_{s}\right)$ values can be found from the measurements of the renormalized $g$-factor: $F_{0}^{\sigma}=\left(g_{b} / g^{*}\right)-1$. Here $r_{s} \equiv 1 / \bar{a}_{B}^{*} \sqrt{\pi n}$ is the dimensionless ratio of the Wigner-Seitz radius to the effective Bohr radius, $\bar{a}_{B}^{*}=\bar{\kappa} / m e^{2}, \bar{\kappa}=7.7$ is the average dielectric constant of $\mathrm{Si}$ and $\mathrm{SiO}_{2}$, and $m=0.19 m_{e}$ is the electron band mass in bulk $\mathrm{Si}^{40.46}$. In our experiments ${ }^{25}$, $g^{*}(n)$ was obtained from the analysis of $\mathrm{SdH}$ oscillations as the ratio of two quantities: the measured renormalized electron spin susceptibility $\chi^{*} / \chi_{b}=g^{*} m^{*} / g_{b} m_{b}$ and the effective mass $m^{*}$ ( $\chi_{b}$ is the bare value of spin susceptibility). Observation of the beatings of $\mathrm{SdH}$ oscillations in crossed magnetic fields offers a straightforward (model-independent) method of finding $g^{*} m^{*} \underline{25}, \underline{43}$. On the other hand, an estimate of $m^{*}$ is based on a model-dependent analysis of the damping factor for the first harmonic of $\mathrm{SdH}$ oscillations, $A_{1}\left(T, B_{\perp}=\right.$ const). According to the LK theory, the damping factor can be expressed as

$$
-\ln \left[A_{1}^{\mathrm{LK}}\left(T, B_{\perp}\right)\right] \frac{B_{\perp}}{2 \pi^{2} m^{*}} \approx\left(T+T_{D}\right)
$$


Our experiments show that $\ln A_{1}\left(T, B_{+}\right)$varies linearly with $T$ within the experimental range $T=(0.4-0.8) \mathrm{K}$ (see Fig. 3 in Ref. 25$)$ ); this however does not prove the applicability of the LK theory, which disregards the interaction effects. It was recently shown $\underline{45}, \underline{47}$ that due to the interference between electron-electron and electron-impurity interactions, the damping factor acquires an additional term in both the diffusive and ballistic regimes

$$
-\ln \left[A_{1}\left(T, B_{\perp}\right)\right] \frac{B_{\perp}}{2 \pi^{2} m^{*}}=\left(T+T_{D}\right)-\alpha(T),
$$

where

$$
\alpha(T)=-T \frac{\delta m^{*}}{m^{*}}-T_{D}\left(\frac{\delta m^{*}}{m^{*}}-\frac{\delta \tau_{D}^{*}}{\tau_{D}^{*}}\right)
$$

and

$$
\begin{aligned}
\frac{\delta m^{*}(T)}{m^{*}} & =-\mathcal{A} \times \ln \left(\frac{E_{F}}{T}\right), \\
\frac{\delta \tau_{D}^{*}(T)}{\tau_{D}^{*}} & =\mathcal{A} \times\left[2 \pi T \tau-\ln \left(\frac{E_{F}}{T}\right)\right], \\
\mathcal{A} & =\left(1+\frac{15 F_{0}^{\sigma}}{1+F_{0}^{\sigma}}\right) \frac{1}{4 \pi^{2} \sigma_{D}},
\end{aligned}
$$

for a system with two degenerate valleys.

The equation for $\delta m^{*}(T) / m^{*}$ resembles the one-loop renormalization of the effective mass (or $Z$ ) in the RG theory $\underline{28}, \underline{29}, \underline{35}$. Our numerical simulations show that within the relevant interval $T=(0.03-0.8) \mathrm{K}$ and $r_{s} \lesssim 6$, the $\ln T$ terms in Eqs. (11) can be replaced with a $T$ independent constant. By combining the LK result with the interaction-induced corrections and replacing all terms $\propto \ln T$ by a constant within our limited $T$ range, we obtain the following linearized equation in the ballistic regime for the short-range scattering $\left(\tau_{D} \sim \tau\right)$ :

$$
-\ln \left[A_{1}\left(T, B_{\perp}\right)\right] \frac{B_{\perp}}{2 \pi^{2} m^{*}}=T+T_{D}(1-2 \pi \mathcal{A} T \tau)=T+T_{D}\left(1-\frac{1}{2} \frac{\delta \sigma(T)}{\sigma_{D}}\right) .
$$

In this case, the $T$-dependent correction to the Dingle temperature, $\frac{\delta T_{D}(T)}{T_{D}}$, is one half of the interaction correction to the conductivity $\frac{\underline{\underline{48}}}{\frac{\delta \sigma(T)}{\sigma_{D}}}$ (this factor $\frac{1}{2}$ originates from the difference between the interaction corrections to the momentum relaxation and quantum scattering times ${ }^{49}$ ). We note that the empirical procedure used for finding $m^{*}$ in our earlier paper (Ref.25) was based on the assumption that $T_{D}^{*}=T_{D}\left[1-\delta \sigma(T) / \sigma_{D}\right]$, which differs from Eq. (12) by a factor of $\frac{1}{2}$.

At relatively high densities (which correspond to $r_{s}<4$ ), the corrections to the LK result are insignificant within the studied $T$-range. As $r_{s}$ increases, the temperature dependences 
of the oscillation magnitude predicted by the LK theory Eq. (8) and the interaction theory $\underline{47}$ start deviating from each other. The values of $\left|F_{0}^{\sigma}\right|$ extracted from $\mathrm{SdH}$ data using Eq. (12) are larger than those obtained with the LK theory but smaller than $\left|F_{0}^{\sigma}\right|$ obtained with

the empirical procedure of Ref. 25 (e.g., at $r_{s}=6.2$, the values of $F_{0}^{\sigma}$ obtained according to Eq. 12 and the empirical procedure of Ref. 25 are -0.40 and -0.45 , respectively). We have reanalyzed the data of Ref.25 using Eq. (12) and compared the corresponding values with $F_{0}^{\sigma}(n)$ extracted from the $\sigma\left(T, B_{\|}\right)$dependences using the ZNA theory (see below).

\section{Valley splitting and intervalley scattering}

The analysis of SdH oscillations using Eq. (7) also allowed us to estimate the energy splitting $\Delta_{V}$ between the valleys. A non-zero valley splitting results in the beating of $\mathrm{SdH}$ oscillations $^{50}$. Figure 1 (a,b) shows the SdH oscillations for samples Si6-14 and Si1-46 (the electron densities are $6.1 \times 10^{11} \mathrm{~cm}^{-2}$ and $1 \times 10^{12} \mathrm{~cm}^{-2}$, respectively). The amplitude of $\mathrm{SdH}$ oscillations normalized by the first harmonic $A_{1}$ is expected to be field-independent if $\Delta_{V}=0$. A noticeable reduction of the $\mathrm{SdH}$ amplitude observed for both samples at small fields can be attributed to a finite valley splitting. Although the node of $\mathrm{SdH}$ oscillations (expected at $B \approx 0.15 \mathrm{~T}$ ) cannot be resolved for samples with mobilities $\mu \cong 2 \mathrm{~m}^{2} / \mathrm{Vs}$, $\Delta_{V}$ can be estimated from fitting of the $B$-dependence of the $\mathrm{SdH}$ amplitude with Eq. (7) modified for the case of a finite $\Delta_{V}: \Delta_{V} \cong 0.4 \mathrm{~K}$ for sample Si6-14 and $0.7 \mathrm{~K}$ for Si1-46. This estimate provides the upper limit for $\Delta_{V}$ at $B=0$ : in nonzero $B_{\perp}$ fields, $\Delta_{V}$ may be enhanced by the inter-level interaction effects $\underline{40,51}$.

The intervalley scattering rate for sample Si6-14 was measured earlier in Ref.52 by analyzing the weak-localization (WL) magnetoresistance. It was found that $\tau_{v}$ is temperatureindependent and the ratio $\tau_{v} / \tau$ decreases monotonically with increasing electron density. For Si6-14 at $n=(3-6) \times 10^{11} \mathrm{~cm}^{-2}, \tau_{v} \cong 20 \mathrm{ps}\left(\tau_{v}^{-1} \cong 0.36 \mathrm{~K}\right)$ is approximately ten times greater than the transport time $\tau \cong 2$ ps.

\section{Relaxation time $\tau$ and the band mass $m_{b}$}

The momentum relaxation time $\tau$ was determined from the Drude conductivity $\sigma_{D}=$ $n \tau / m_{b}$, which was found by extrapolating the quasi-linear $\sigma(T)$ dependence observed in 
the ballistic regime to $T=0$. Note that in order to extract $\tau$ from the Drude conductivity, one should use the bare mass $m_{b}$ : according to the Kohn theorem, the response of a translationally-invariant system to the electromagnetic field is described by $m_{b}$ in the presence of electron-electron interactions; this result also holds for weak disorder $\left(E_{F} \tau \gg 1\right)$. It is worth mentioning that several prior publications $36,37,38$, including our paper $\underline{37}$, incorrectly used $m^{*}$ instead of $m_{b}$ to estimate $\tau$ from $\sigma_{D}$; this affects the value of the fitting parameters extracted from comparison with the theory ${ }^{26}$ as shown below.

The textbook value ${ }^{40}$ for the light electron mass in bulk $\mathrm{Si}$ is $m_{b}^{(3 D)} \approx 0.19 m_{e}$. For inversion layers on (001) Si-surface, Kunze and Lautz $\underline{53}$ have obtained $m_{b}^{(2 D)} / m_{e}=(0.19-$ $0.22) \pm 0.02$ from tunneling measurements. Our recent $m^{*}(n)$ data obtained from the analysis of $\mathrm{SdH}$ oscillations ${ }^{25}$ over a wide range of densities $r_{s}=1.4-8.5$, can be fitted with a polynomial $m^{*}\left(r_{s}\right)=0.205 m_{e}\left(1+0.035 r_{s}+0.00025 r_{s}^{4}\right)$. These $m^{*} / m_{e}$ data agree well with earlier values of $m^{*}$ extracted from $\mathrm{SdH}$ oscillations $54,55,56,57$ in narrower ranges of densities. By extrapolating the polynomial $m^{*}\left(r_{s}\right)$ to $r_{s}=0$ we obtain $m_{b}^{(2 D)} / m_{e}=0.205 \pm 0.005$, the value which we adopted throughout the paper ${ }^{58}$; available measurements of the cyclotron resonance ${ }^{59}$ do not contradict and do not refine this value.

In principle, the aforementioned complete characterization of samples allows us to compare the $\Delta \sigma(T, B)$ dependences with the theory ${ }^{26}$ without any fitting parameters (with a caveat that the theory does not take into account the intervalley scattering, see the discussion below). However, throughout this paper we adopt an equivalent, but more convenient procedure: for each electron density, $F_{0}^{\sigma}(n)$ will be considered as a single parameter for fitting $\Delta \sigma(T, B)$, and these values of $F_{0}^{\sigma}$ will be compared with the corresponding values obtained from the $\mathrm{SdH}$ oscillations 25 .

\section{B. Temperature dependences of the conductivity at $B_{\|}=0$}

The temperature dependences of the conductivity $\sigma(T)$ for sample Si6-14 are shown in Fig. 2. In these measurements, we applied a fixed $B_{\perp}=0.1 \mathrm{~T}$ that is sufficient to suppress the temperature dependence of the WL correction in the studied temperature range. The $\sigma(T)$ dependences are non-monotonic for all studied densities $\left[n=(1.8-15) \times 10^{11} \mathrm{~cm}^{-2}\right.$ for Si6-14 and $n=(10-15) \times 10^{11} \mathrm{~cm}^{-2}$ for Si1-46]: a quasi-linear increase of $\sigma$ with cooling, observed down to $\sim 0.5 \mathrm{~K}$, is replaced at lower $T$ with a decrease of $\sigma$. Note that in our previous 
$\operatorname{experiments}^{37}$, we observed a trend of $\sigma(T)$ saturation at $T<0.4 \mathrm{~K}$ rather than the decrease of the conductivity. One of the reasons for this might have been "heating" of electrons by high frequency noise: only after thorough filtering of all leads connected to the sample were we able to decrease the electron temperature down to $\sim 30 \mathrm{mK}$. Similar downturn of $\sigma(T)$, although at much lower temperatures, has been recently observed in high- $\mu$ GaAs FETs at low electron densities 60 . We note also that earlier, a downturn of $\sigma(T)$ was observed in Si-MOSFETs ${ }^{61}$ but at much higher electron densities $\left(n>30 \times 10^{11} \mathrm{~cm}^{-2}, r_{s}<1.4\right)$ and at much higher temperatures $T \sim 10 \mathrm{~K}(T \tau \approx 1)$. For such high densities the interaction corrections to $\sigma$ become negative and the downturn of $\sigma(T)$ was related to the crossover between a "metallic" high-temperature $\sigma(T)$ dependence (that is due to electron-phonon and intersubband scattering effects) and resulting "insulating" low-temperature $\sigma(T)$ dependence (that is due to weak localization and negative interaction corrections contributions to $\sigma(T)$ ).

Below we use the following strategy for analyzing the $\Delta \sigma(T)$ dependences. First, we find $F_{0}^{\sigma}$ by fitting the quasi-linear $\sigma(T)$ dependences observed in the ballistic regime $(T>0.5 \mathrm{~K})$ with Eqs. (11-3). The effect of valley splitting and intervalley scattering on $\Delta \sigma_{e e}(T)$ can be neglected at $T \gg \Delta_{V}, \tau_{v}^{-1}$ and the analysis is significantly simplified. The corresponding values of $F_{0}^{\sigma}(n)$ are shown in Fig. 5. The $T$ range available for fitting in this regime "shrinks" rapidly at low $n$ : the growth of $\left|F_{0}^{\sigma}\right|$ and decrease of $E_{F}$ with decreasing $n$, lead to violation of the condition $T \ll\left(1+F_{0}^{\sigma}\right)^{2} E_{F}$ (e.g., at $n=1.8 \times 10^{11} \mathrm{~cm}^{-2}$ this occurs at temperatures above $2 \mathrm{~K}$ ). This might be one of the reasons for the observed deviation of the high-temperature $\sigma(T)$ from the linear-in- $\left(T / E_{F}\right)$ theory ${ }^{26}$. Also, the higher-order corrections might become significant at low $n$ when $\Delta \sigma(T) / \sigma_{D} \sim 1$ (see Fig. 2).

After finding the $F_{0}^{\sigma}$ values (which are temperature-independent in the studied temperature range), we proceed with the analysis of the low $-T$ part of the $\sigma(T)$ dependences, where the crossover from $d \sigma / d T<0$ to $d \sigma / d T>0$ was observed. We note that the crossover occurs when the temperature becomes smaller than two characteristic temperature scales $-\Delta_{V}$ and $\tau_{v}^{-1}$ - which are of the same order of magnitude for the studied structures. We emphasize that according to the ZNA theory ${ }^{26}$, the ballistic-diffusive crossover should not lead to the change of the sign of $d \sigma / d T$. In contrast, the valley splitting and the intervalley scattering may result in the sign change for $d \sigma / d T$ because these processes reduce the number of triplet components at $T<\Delta_{V}$ and $T<\tau_{v}^{-1}$.

The theory ${ }^{26}$ takes into account a finite $\Delta_{V}$ but not $\tau_{v}^{-1}$. The solid red curves in Fig. 2 
are calculated for $\Delta_{V}=0.4 \mathrm{~K}$ (the estimated value of $\Delta_{V}$ for sample Si6-14) and $\tau_{v}^{-1}=0$. It is clear that the change in the number of triplet components from $15\left(T \gg \Delta_{V}\right)$ to 7 $\left(T \ll \Delta_{v}\right)^{38,62}$ (see also Eq. 51) is not sufficient to explain the shape of the $\sigma(T)$ downturn. The effect of strong intervalley scattering is illustrated in Fig. 2 by dashed green curves calculated with 3 triplet components (to model roughly the case of $T \ll \tau_{v}^{-1}$ when the valleys are completely intermixed). In the absence of a detailed theory that would account for intervalley mixing, we attempted to fit the experimental data with an empirical crossover function for the number of triplet components $N_{\text {triplet }}(x)=9+6[\exp (-0.3 / x)-\exp (-30 x)]$, where $x=T \tau_{v}$. This crossover function provides correct asymptotic limits for $N_{\text {triplet }}: 3$ at $T \ll \tau_{v}^{-1}$ and 15 at $T \gg \tau_{v}^{-1}$. Figure 2 shows that using this function, we can reasonably well describe the shape of experimental $\sigma(T)$ dependences for all studied electron densities.

\section{Temperature dependences of the conductivity at non-zero $B_{\|}$}

Better understanding of different contributions to $\Delta \sigma(T)$ can be achieved by measuring the conductivity in strong in-plane magnetic fields $B_{\|} \gg T / g_{b} \mu_{B}$. The evolution of experimental dependences $\sigma(T)$ with $B_{\|}$is shown in Fig. 3 for two samples at different electron densities. The theoretical curves in Fig. 3 were calculated using the $F_{0}^{\sigma}(n)$ values extracted from the analysis of $\delta \sigma_{e e}\left(T, B_{\|}=0\right)$ (see Fig. 2). The transport time $\tau$ was calculated for each $B_{\|}$value from the Drude conductivity $\sigma_{D}\left(B_{\|}\right)$which in turn was estimated by extrapolating the quasi-linear part of the $\sigma\left(T, B_{\|}\right)$dependence to $T=0$. The observed behavior is in line with our analysis of the $\sigma(T)$ dependences in Section III B. Indeed, the magnitude of the triplet contribution is expected to be reduced when the Zeeman energy becomes greater than $T$. This effect is more pronounced within the range $\Delta_{V}, \tau_{v}^{-1}<T<g_{b} \mu_{B} B$, where a strong magnetic field reduces the number of triplet components from 15 to 7. For example, at $n=1 \times 10^{12} \mathrm{~cm}^{-2}$ (see Fig. $3 \mathrm{~d}$ ) the "metallic" behavior disappears at $T<1 \mathrm{~K}$ and $B_{\|}=3 \mathrm{~T}$, which is in agreement with the theory. At lower $T$, the number of triplet components is smaller than 15 even at $B_{\|}=0$ due to valley splitting and intervalley scattering, and the effect of $B_{\|}$on $\Delta \sigma(T)$ is less prominent. 


\section{Magnetoconductivity}

To test the theoretical predictions on the magnetoconductivity (MC) induced by in-plane magnetic fields, we also measured the $\sigma\left(B_{\|}\right)$dependences at fixed $T$. Similar measurements have been performed in the past (see, e.g., Refs. 37,38,63), but no detailed comparison with the theory was carried out at that time. The MC for sample Si6-14 over the field range $-4.5<B_{\|}<4.5 \mathrm{~T}$ is shown for different densities and temperatures in Fig. 4 . In these measurements, special care was taken to reduce the magnetic field component perpendicular to the plane of the structure: even a $1^{\circ}$ misalignment between the sample's plane and the magnet axis (which results in $B_{\perp} \sim 50 \mathrm{G}$ at $B_{\|}=3 \mathrm{~T}$ ) may be sufficiently strong for suppressing the WL corrections at low $T$. To eliminate $B_{\perp}$, we used the cross-magnetic-field set-up $\stackrel{43}{\text {. }}$ For each value of $B_{\|}$, we measured the dependence $\sigma\left(B_{\perp}\right)$ by sweeping $B_{\perp}$ and recorded the minimum value of $\sigma\left(B_{\perp}\right)$ which corresponded to the zero WL magnetoconductance and, thus, $B_{\perp}=0$. This method allowed us to compensate $B_{\perp}$ with an accuracy better than $10 \mathrm{G}$.

The theoretical $\Delta \sigma\left(B_{\|}\right)$dependences (see Eqs. (56) ), plotted in Fig. 4 as solid curves, describe the observed MC very well in not-too-strong magnetic fields $g_{b} \mu_{B} B_{\|}<0.2 E_{F}$. Again, as in the case of fitting the $\Delta \sigma(T)$ dependences, the only adjustable parameter was the $F_{0}^{\sigma}(n)$ value extracted for each density from fitting the $\mathrm{MC}$ at high temperatures $(\approx 0.7 \mathrm{~K})$ where the effects of valley splitting or intervalley scattering on $\Delta \sigma_{e e}(T, B)$ can be neglected. Note that all the theoretical curves plotted in Fig. 4 for the same $n$ were calculated for a fixed $F_{0}^{\sigma}(n)$, i.e. neglecting possible dependence $F_{0}^{\sigma}\left(B_{\|}\right)$. The detailed analysis of the spin susceptibility $\chi^{*} \propto g^{*} m^{*}$ in strong magnetic field, presented in Ref.64, shows that the product $g^{*} m^{*}$ decreases with an increase of $B_{\|}$by as much as $\sim 20 \%$. Our estimate shows that by ignoring the $g^{*}(B)$ dependence, we might reduce the value of $\left|F_{0}^{\sigma}\right|$ by $\sim 10 \%$ (see below), which is close to the accuracy of extraction of $F_{0}^{\sigma}$ from the data in strong magnetic fields. As $B_{\|}$grows and/or $n$ decreases, the data start deviating from the theoretical curves (see Fig. $4 \mathrm{~d}$ ); this deviation can be attributed to the violation of the condition $g_{b} \mu_{B} B \ll E_{F}$ required for applicability of Eqs. (55,6). 


\section{E. The $F_{0}^{\sigma}(n)$ dependence}

The $F_{0}^{\sigma}$ values obtained from fitting the $\Delta \sigma(T)$ and $\Delta \sigma\left(B_{\|}\right)$dependences with the theory $\underline{26}$ are shown in Fig. 5. For comparison, we have also plotted the $F_{0}^{\sigma}$ values obtained from the analysis of SdH oscillations measured for sample Si6-14 using the theories $\underline{44}$.47 (see Section III A). The $F_{0}^{\sigma}$ values obtained from fitting $\Delta \sigma(T)$ are in good agreement with the corresponding values extracted from the analysis of SdH data.

At the same time, the $\left|F_{0}^{\sigma}\right|$ values obtained from fitting the $\Delta \sigma\left(B_{\|}\right)$dependences at $r_{s}>4$ are systematically smaller than the corresponding values obtained from fitting $\Delta \sigma(T)$ and SdH oscillations. This trend was earlier reported in Refs. 37,38. There are at least two factors that can reduce this discrepancy. One of them, a potential decrease of $g^{*}$ in strong $B_{\|}$, was mentioned in Section III D. The other factor is more subtle. In our analysis, we neglected the dependence of the WL correction $\delta \sigma_{\mathrm{WL}}$ on $B_{\|}$. However, our measurements show that $\delta \sigma_{\mathrm{WL}}$ decreases with an increase of the in-plane magnetic field, which leads to a positive magnetoconductance. There are at least two potential reasons for this dependence: (a) the $\mathrm{Si}-\mathrm{SiO}_{2}$ interface roughness transforms a uniform in-plane field into a random perpendicular field (see, e.g., Refs. 65, 66, 67 and references therein), and (b) a finite extent of electron wave functions in the direction perpendicular to the plane of a quantum well causes sub-band mixing by the magnetic field and disorder (see, e.g., Ref.68 and references therein).

Phenomenologically, both effects can be described in terms of a decrease of the dephasing length $L_{\varphi}$ with $B_{\|}$. For example, from the analysis of the WL magnetoresistance measured for different values of $B_{\|}$for sample Si6-14 at $n=1 \times 10^{12} \mathrm{~cm}^{-2}$ and $T=0.3 \mathrm{~K}$, we have extracted $L_{\varphi}\left(B_{\|}=0\right)=1.3 \mu \mathrm{m}$ and $L_{\varphi}\left(B_{\|}=3 T\right)=0.8 \mu \mathrm{m}$. Our estimates show that the positive magnetoconductance associated with the suppression of $\delta \sigma_{\mathrm{WL}}$ by $B_{\|}$can account for $\sim 10 \%$ of the observed $B_{\|}$-induced magnetoconductance. After taking the dependences $g^{*}\left(B_{\|}\right)$and $\delta \sigma_{\mathrm{WL}}\left(B_{\|}\right)$into account, the $\left|F_{0}^{\sigma}\right|$ values extracted from the MC should be increased by $\sim 20 \%$. The corresponding downshift of the $F_{0}^{\sigma}$ values extracted from the $\Delta \sigma(B)$ data is shown by arrows in Fig. $5 \mathrm{a}$, it significantly reduces the discrepancy between the values of $F_{0}^{\sigma}$ extracted from the $B_{\|}=0$ data and the data measured at $B_{\|}=1-5 \mathrm{~T}$.

Finally, in order to compare our data with other available $\Delta \sigma(T)$ data for high-mobility Si MOSFETs, we have used Eqs. (16) to estimate the $F_{0}^{\sigma}$ values from the quasi-linear $\sigma(T, B=0)$ dependences measured by Shashkin et al $\frac{36}{\underline{36}}$ and Vitkalov et al. $\underline{\underline{38}}$. In this 
analysis, we estimated $\tau$ from $\sigma(T \rightarrow 0)$ using the band mass rather than $m^{*}$. As for the valley splitting, we have used $\Delta_{V}=0.6 \mathrm{~K}$ and $1 \mathrm{~K}$ for the analysis of data from Ref. 36 and 38, respectively. (The value $\Delta_{V} \sim(1-2) \mathrm{K}$ used in Ref.36 seems to be too large, as it would lead to the appearance of beating of $\mathrm{SdH}$ oscillations in the field range studied in Ref.69). We have also taken into account the WL correction neglected in both Ref.36 and 38. Figure $5 \mathrm{~b}$ shows that the $F_{0}^{\sigma}\left(r_{s}\right)$ values estimated for different Si MOSFETs using the ZNA theory are in good agreement with each other.

The $F_{0}^{\sigma}$ values obtained on the basis of the interaction correction theory and plotted in Fig. 5 may be compared also with the values of $F_{0}^{\sigma}=-\gamma_{2} /\left(1+\gamma_{2}\right)$ predicted by the RG theory and measured experimentally in Refs. 31, 32. Extrapolation of our $F_{0}^{\sigma}\left(r_{s}\right)$ data to lower densities, provides the value $F_{0}^{\sigma} \approx-0.5$ at $n \approx 1 \times 10^{11} \mathrm{~cm}^{-2}\left(r_{s} \approx 8\right)$, which is smaller than the value $F_{0}^{\sigma}=-0.31\left(\gamma_{2}=0.45\right)$ predicted by the one-loop RG theory

for the temperature $T^{\max }$ corresponding to the $\rho(T)$ maximum 29 , 31,32 (e.g., $T^{\max } \approx 3 \mathrm{~K}$ for $\left.n=1.2 \times 10^{11} \mathrm{~cm}^{-2}\right)$.

The experimental test $\stackrel{31,32}{ }$ of the RG theory was conducted at temperatures higher than that in the experiments described in the present work. Within the framework of the RG theory, the interaction parameter $\gamma_{2}$ is expected to increases with decreasing $T^{29,31,32}$ and, in principle, it can reach at $T<1 K$ the value of $\sim 1$ which corresponds to $F_{0}^{\sigma}=-0.5$. (Note that the factor-of-two increase of $\gamma_{2}$ (from 0.45 to 1 ) is beyond the range of the applicability of the one-loop RG theory.) Another problem is that the spin susceptibility $\chi^{*} \propto g^{*} m^{*}$ obtained from the $\mathrm{SdH}$ data is almost $T$-independent $\underline{64}$, in contrast to the expected increase of $\gamma_{2}$ (and, hence, $\left|F_{0}^{\sigma}\right|$ and $g^{*}$ ) with cooling. This contradiction can be resolved if the $T$-dependence of $g^{*}$ is exactly compensated by the opposite $T$-dependence of $m^{*}$, so that $\chi^{*} \propto g^{*} m^{*}$ remains almost constant. The reason for this compensation is not clear and requires both experimental and theoretical studies.

\section{CONCLUSION}

Our experiments show that the low- $T$ behavior of the conductivity of high-mobility (001) Si MOSFETs is well described by the theory of interaction effects in systems with short-range disorder $\underline{26}$. Over a wide range of intermediate temperatures $\left(\Delta_{V}, \tau_{v}^{-1}, g_{b} \mu_{B} B<\right.$ $\left.T \ll E_{F}\right)$, the interaction effects are strongly enhanced in Si MOSFETs due to the presence 
of two valleys in the electron spectrum. This factor, in combination with the interactiondriven renormalization of the Fermi-liquid parameter $F_{0}^{\sigma}$, leads to an increase of $\sigma$ with decreasing $T$. At lower temperatures $\left(T<\Delta_{V}, \tau_{v}^{-1}, g_{b} \mu_{B} B, E_{F}\right)$, the triplet contribution to $\Delta \sigma_{e e}(T)$ is significantly reduced due to valley splitting and/or intervalley scattering. As a result, the "metallic" behavior of $\sigma$ is replaced with a more conventional, "insulating" behavior. The $F_{0}^{\sigma}$ values obtained from fitting the experimental data with the theory $\underline{26}^{2}$ agree well with the $F_{0}^{\sigma}$ data obtained from the analysis of $\mathrm{SdH}$ oscillations in these samples. However, it remains unclear how to reconcile the $F_{0}^{\sigma}$ values obtained at low $n$ from fitting the $\sigma(T)$ and $\mathrm{SdH}$ data by using the interaction correction theory with the corresponding values obtained within framework of the RG theory.

We emphasize that for the detailed analysis of the interaction-induced contributions to the conductivity, it is important to measure such parameters as the valley splitting and intervalley scattering rate in independent experiments. Finally, for a quantitative description of the interaction effects to the conductivity $\Delta \sigma_{e e}$ at low temperatures, both the interaction correction theory and RG theory should be extended to the case of a finite intervalley scattering rate.

\section{ACKNOWLEDGMENTS}

The authors are thankful to E. Abrahams, I. Aleiner, I. Burmistrov, I. Gornyi, G. Kotliar, and A. Mirlin for illuminating discussions. The research at Rutgers University was partially supported by the NSF grant ECE-0608842. The research at Lebedev Institute was supported by RFBR, Programs of the RAS, Russian Ministry for Education and Science, and the Program "Leading Scientific Schools".

1 E. Abrahams, P. W. Anderson, D. C. Licceardello, and T. V. Ramakrishnan, Phys. Rev. Lett. 42, 673 (1979).

2 F. J. Wegner, Phys. Rev. B 19, 783 (1979).

3 B. L. Altshuler, A. G. Aronov, and P. A. Lee, Phys. Rev. Lett. 44, 1288 (1980); B. L. Altshuler and A. G. Aronov, in Electron-Electron Interaction in Disordered Systems, edited by A. L. Efros and M. Pollak (North-Holland, Amsterdam, 1985). 
4 B. L. Altshuler, A. G. Aronov, M.E. Gershenson, and Yu. V. Sharvin, Sov. Sci. Rev. A. Phys. 9, 223-254 (1987).

5 D. J. Bishop, D. C. Tsui, and R. C. Dynes, Phys. Rev. Lett. 44, 1153 (1980); Phys. Rev. B 26, $773(1982)$.

6 V. T. Dolgopolov, S. I. Dorozhkin, A. A. Shashkin, Sol. State Commun. 50, 273 (1984).

7 L. P. Gorkov, A. I. Larkin, and D. E. Khmelnitskii, JETP Lett., 30, 228 (1979). [Pis'ma v Zh. Eksp. Teor. Fiz. 30, 248 (1979)].

8 M. S. Burdis and C. C. Dean, Phys. Rev. B 38, 3269 (1988).

9 K. M. Cham and R. G. Wheeler, Phys. Rev. Lett. 44, 1472 (1980).

10 R. P. Smith and P. J. Stiles, Sol. State Commun. 58, 511 (1986).

11 T. N. Zavaritskaya and E. I. Zavaritskaya, JETP Lett. 45, 609 (1987). [Pis'ma v Zh. Eksp. Teor. Fiz. 45, 476 (1987)].

12 E. A. Virodov, V. T. Dolgopolov, S. I. Dorozhkin, N. B. Zhitenev, Zh. Eksp. Teor. Fiz. 94, 234; [JETP 67, 998 (1988)].

13 S. V. Kravchenko, G. V. Kravchenko, J. E. Furneaux, V. M. Pudalov, and M. D’Iorio, Phys. Rev. B 50, 8039 (1994).

14 Y. Hanein, U. Meirav, D. Shahar, C. C. Li, D. C. Tsui, and H. Shtrikman, Phys. Rev. Lett. 80, 1288 (1998); Y. Hanein, D. Shahar, J. Yoon, C. C. Li, D. C. Tsui, and H. Shtriktman, Phys. Rev. B 56, R7520 (1998); A. R. Hamilton, M. Y. Simmons, M. Pepper, E. H. Linfield, P. D. Rose, and D. A. Ritchie, Phys. Rev. Lett. 82, 1542 (1999); A. P. Mills, A. P. Ramirez, L. N. Pfeiffer, and K. West, Phys. Rev. Lett. 83, 2805 (1999).

15 M. Y. Simmons, A.R. Hamilton, M. Pepper, E. H. Linfield, P. D. Rose, D. A. Ritchie, A. K. Savchenko, and T.G. Griffiths, Phys. Rev. Lett. 80, 1292, (1998); J. Yoon, C. C. Li, D. Shahar, D. C. Tsui, and M. Shayegan, Phys. Rev. Lett. 82, 1744 (1999).

16 S. J. Papadakis, E.P. De Poortere, H.C. Manoharan, M. Shayegan, and R. Winkler, Science 283, 2056 (1999).

17 Y. Hanein, D. Shahar, H. Shtrikman, J. Yoon, C. C. Li, and D. C. Tsui, Nature 400, 735 (1999).

18 Y. Hanein, D. Shahar, J. Yoon, C. C. Li, D. C. Tsui, and H. Shtrikman, Phys. Rev. B 58, R13338 (1998).

19 E. Ribeiro, R. D. Jäggi, T. Heinzel, K. Ensslin, G. Medeiros-Ribeiro, and P. M. Petroff, Phys. Rev. Lett. 82, 996 (1999). 
20 P. T. Coleridge, R. L. Williams, Y. Feng, and P. Zawadzki, Phys. Rev. B 56, R12764 (1997); J. Lam, M. D’Iorio, D. Brown, and H. Lafontaine, Phys. Rev. B 56, R12741 (1997); P. T. Coleridge, A. Sachrajda, and P. Zawadzki, Phys. Rev. B 65, 125328 (2002).

21 S. J. Papadakis and M. Shayegan, Phys. Rev. B 57, R15068 (1998).

22 F. Stern and W. E. Howard, Phys. Rev. 163, 816 (1963); A. Gold and V. T. Dolgopolov, Phys. Rev. B 33, 1076 (1986); S. Das Sarma, Phys. Rev. B 33, 5401 (1986); S. Das Sarma, and E. H. Hwang, Phys. Rev. Lett. 83, 164 (1999); S. Das Sarma, and E. H. Hwang, Phys. Rev. B 69, 195305 (2004).

23 D. Simonian S. V. Kravchenko, M. P. Sarachik, and V. M. Pudalov, Phys. Rev. Lett. 79, 2304 (1997); V. M. Pudalov, G. Brunthaler, A. Prinz, G. Bauer, JETP Lett. 65, 933 (1997).

24 T. Okamoto, K. Hosoya, S. Kawaji, and A. Yagi, Phys. Rev. Lett. 82, 3875 (1999).

25 V. M. Pudalov, M. E. Gershenson, H. Kojima, N. Butch, E. M. Dizhur, G. Brunthaler, A. Prinz, and G. Bauer, Phys. Rev. Lett. 88, 196404 (2002).

26 G. Zala, B. N. Narozhny, and I. L. Aleiner, Phys. Rev. B 64, 214204 (2001); ibid 65, 020201 (2002).

27 I. V. Gornyi and A. D. Mirlin, Phys. Rev. B. 69, 045313 (2004).

28 A. M. Finkel'stein, Z. Phys. B 56189 (1984); Sov. Sci. Rev. A, Phys. 14, 1-100, (1990).

29 A. Punnoose and A. M. Finkel'stein, Phys. Rev. Lett. 8816802 (2002).

30 A. Punnoose and A. M. Finkel'stein, Science 310, 289 (2005).

31 D. A. Knyazev, O. E. Omel'yanovskii, V. M. Pudalov, and I. S. Burmistrov, JETP Lett. 84, 662 (2006).

32 S. Anissimova, S. V. Kravchenko, A. Punnoose, A. M. Finkel'stein, and T. M. Klapwijk, Nature Phys. 3, 707 (2007).

33 D. A. Knyazev, O. E. Omel'yanovskii, V. M. Pudalov, I. S. Burmistrov, Phys. Rev. Lett. 100, 046405 (2008).

34 C. Castellani, C. Di Castro, P. A. Lee, and M. Ma, Phys. Rev. B 30, 527 (1984).

35 C. Castellani and C. Di Castro, and P. A. Lee, Phys. Rev. B 57, 9381 (1998).

36 A. A. Shashkin, S. V. Kravchenko, V.T. Dolgopolov, and T. M. Klapwijk, Phys. Rev. B 66, $073303(2002)$.

37 V.M. Pudalov, M.E. Gershenson, H. Kojima, G. Brunthaler, A. Prinz, and G. Bauer, Phys. Rev. Lett. 91, 126403 (2003). 

67, $113310(2003)$.

39 W. R. Clarke, C. E. Yasin, A. R. Hamilton, A. P. Micolich, M. Y. Simmons, K. Muraki, Y. Hirayama, M. Pepper and D. A. Ritchie, Nature Physics 4, 55 (2007).

40 T. Ando, A. B. Fowler, and F. Stern, Rev. Mod. Phys. 54, 437 (1982).

41 I. S. Burmistrov and N. M. Chtchelkatchev, JETP Lett. 84, 558 (2006); Phys. Rev. B 77, 195319 (2008).

42 O. Prus, M. Reznikov, U. Sivan, and V. M. Pudalov, Phys. Rev. Lett. 88, 016801 (2002).

43 M. E. Gershenson, V. M. Pudalov, H. Kojima, N. Butch, G. Bauer, G. Brunthaler, and A. Prinz, Physica E 12, 585 (2002).

44 I. M. Lifshitz, A. M. Kosevich, Zh. Eksp. Teor. Fiz. 29, 730 (1955); A. Isihara and L. Smrćka, J. Phys. C 19, 6777 (1986).

45 G. W. Martin, D. L. Maslov, and M. Yu. Reizer, Phys. Rev. B 68, 241309(R) (2003).

46 Here we used a traditional value of $m_{b}$ in order to fasilitate the comparison with the results of other experimental works.

47 Y. Adamov, I. V. Gornyi, and A. D. Mirlin, Phys. Rev. B 73, 045426 (2006).

48 Interaction correction to the conductivity [Eqs. (1-3)] in the ballistic regime has the form $\delta \sigma(T) / \sigma_{D}=T \tau\left[1+15 F_{0}^{\sigma} /\left(1+F_{0}^{\sigma}\right)\right] / \pi \sigma_{D}$.

49 The quantum correction to the transport scattering rate $\tau^{-1}$ differs from that for the quantum scattering rate $\tau_{D}^{-1}$ by the term $(1-\cos (\phi))$ in the integrand, where $\phi$ is the scattering angle. According to ZNA theory ${ }^{26}$, the interaction corrections to the conductivity are determined by backscattering events for which $\phi \approx \pi$, or $(1-\cos (\phi)) \approx 2$.

50 V. M. Pudalov, A. Punnoose, G. Brunthaler, A. Prinz, G. Bauer, cond-mat/0104347. In this paper, three Si MOSFET samples with anomalously large values $\Delta_{V} \sim 1-2 \mathrm{~K}$ were studied; the high $\Delta_{V}$ values enabled observation of the $\mathrm{SdH}$ oscillation beats in low magnetic fileds.

51 V. M. Pudalov, S. G. Semenchinskii, V.S. Edelman, Pis'ma v ZhETF, 41(6), 265 (1985).[JETP Lett. 41, 325 (1985)].

52 A. Yu. Kuntsevich, N. N. Klimov, S. A. Tarasenko, N. S. Averkiev, V. M. Pudalov, H. Kojima, and M. E. Gershenson, Phys. Rev. B 75, 195330 (2007).

53 U. Kunze, G. Lautz, Surface Sci. 142, 314 (1984).

54 F. F. Fang, A. B. Fowler, and A. Hartstein, Phys. Rev. B 16, 4446 (1977). 
55 A. B. Fowler, F. Fang, W. E. Howard, and P. J. Stiles, Phys.Rev. Lett., 16, 901 (1966).

56 F. Fang and P. J. Stiles, Phys. Rev. 174, 823 (1968).

57 J. L. Smith and P. J. Stiles, Phys. Rev. Lett., 29, 102 (1972).

58 Our revision of the analysis of $\mathrm{SdH}$ data (described in the subsection IIIA 1 ) modifies the $m^{*}$ values only at low densities $\left(r_{s}>4\right)$ and does not affect $m^{*}\left(r_{s} \rightarrow 0\right)=0.205 m_{e}$, adopted as the best estimate for $m_{b}$.

59 The chosen value $m_{b}=0.205 m_{e}$ does not contradict the cyclotron resonance data. Due to the Kohn theorem, the mass measured in the cyclotron resonance experiments is believed to be unrenormalized and equal to the band mass (and hence, independent of density). Abstreiter et al. [Phys. Rev. B 14, 2480 (1976)] observed the far-infrared cyclotron resonance and obtained $m_{c}=(0.197 \pm 0.005) m_{e}$ independent of $n$ for $n>1 \times 10^{12} \mathrm{~cm}^{-2}$. However, for $n<1 \times 10^{12} \mathrm{~cm}^{-2}$, they observed a sample-dependent noticeable increase of $m_{c}$ with decreasing density (up to $0.215 m_{e}$ at $n=5 \times 10^{11} \mathrm{~cm}^{-2}$ ), which was attributed to the localization effects.

60 J. Huang, J. S. Xia, D. C. Tsui, L. N. Pfeiffer, and K. W. West, Phys. Rev. Lett. 98, 226801 (2007).

61 V.M.Pudalov, G. Brunthaler, A. Prinz, and G. Bauer, Phys. Rev. B 60 R2154 (1999).

62 For a two-valley system with $\Delta_{v} \gg T$, the total number of interaction channels is $4 \times 2=8$, among them 1 singlet and 7 triplet terms.

63 X.P. A. Gao, A.P. Mills, Jr., A.P. Ramirez, L. N. Pfeiffer, and K. W. West, Phys. Rev. Lett. 88, $166803(2002)$.

64 V. M. Pudalov, M. Gershenson, and H. Kojima, "On the electron-electron interactions in two dimensions", in: Fundamental Problems of Mesoscopic Physics, eds. I. V. Lerner et al. (Kluwer 2004), pp. 309-327.

65 P. M. Mensz and R. G. Wheeler, Phys. Rev. B 35, 2844 (1987).

66 H. Mathur and H. U. Baranger, Phys. Rev. B 64, 235325 (2001).

67 G. M. Minkov et al., Phys. Rev. B 70, 035304 (2004).

68 J. S. Meyer, V. I. Fal'ko, and B. L. Altshuler, in NATO Science Series II, vol. 72, eds. I. V. Lerner et al. (Kluwer Academics, Dordrecht, 2002), pp. 117-164.

69 S. V. Kravchenko et al., Solid State Comm. 116, 495-499 (2000). 

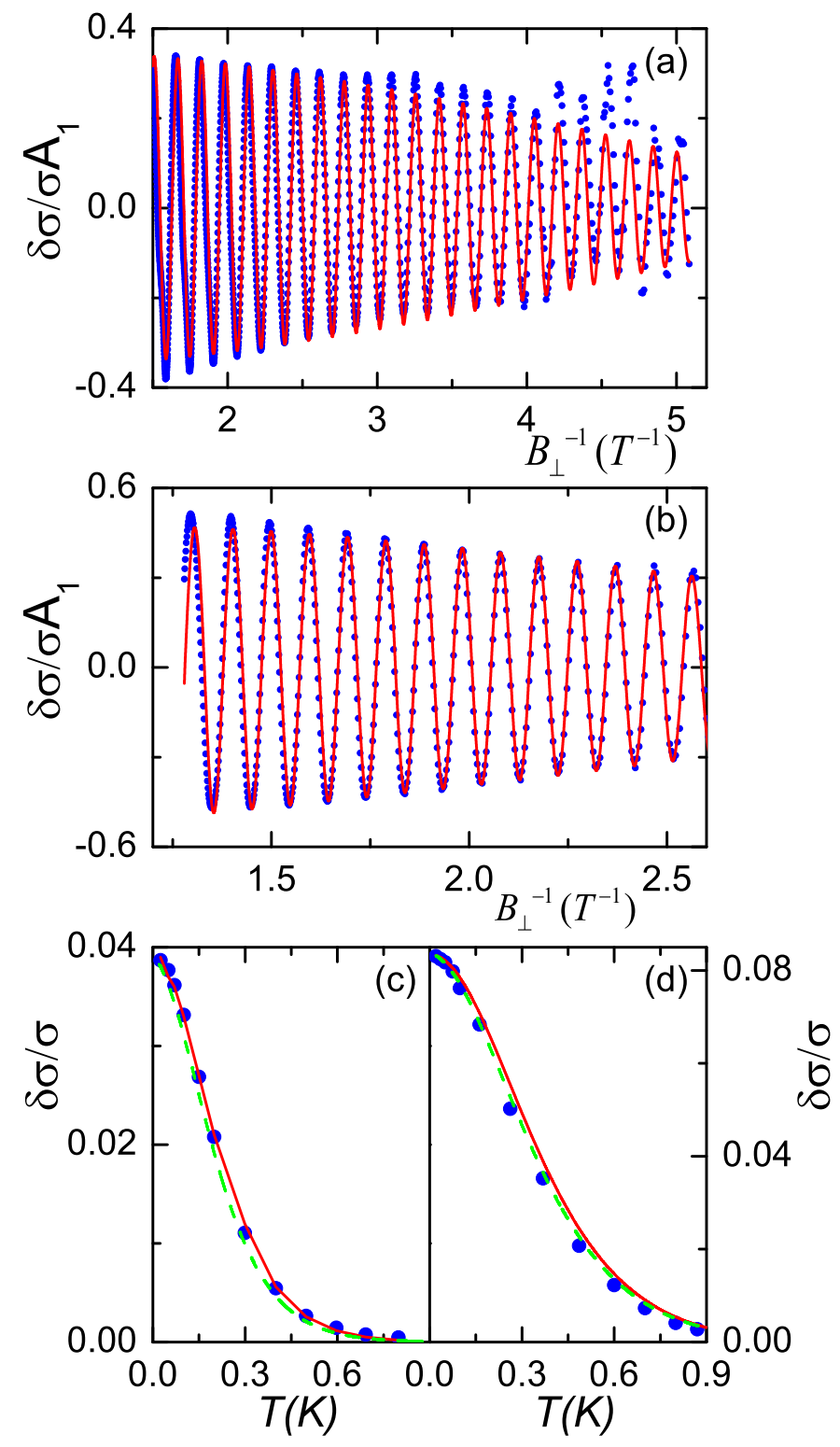

FIG. 1: (color online) Shubnikov - de Haas oscillations normalized by the amplitude of the first harmonic $A_{1}$ : (a) sample Si6-14, $n=6.1 \times 10^{11} \mathrm{~cm}^{-2}, T=36 \mathrm{mK}$; (b) sample Si1-46, $n=1 \times 10^{12} \mathrm{~cm}^{-2}$, $T=200 \mathrm{mK}$. Dots represent the experimental data, solid curves - the theoretical dependences (7) modified for a finite $\Delta_{V}$ and calculated for $\Delta_{V}=0.4 \mathrm{~K}$ and $0.7 \mathrm{~K}$ for samples Si6-14 and Si146 respectively. Panels (c) and (d) show the temperature dependences of the amplitude of SdH oscillations for Si6-14 $\left(n=5.5 \times 10^{11} \mathrm{~cm}^{-2}\right)$ and Si1-46 $\left(n=1 \times 10^{12} \mathrm{~cm}^{-2}\right)$, solid curves - the noninteracting LK-model [Eqs. (8)], dashed curves - the fit based on the interaction theory [Eqs. (9), 10. (11)]. The linearized Eq. (12) is indistinguishable from Eqs. (9, 10, 11) within the studied $T$ range and not shown therefore. 

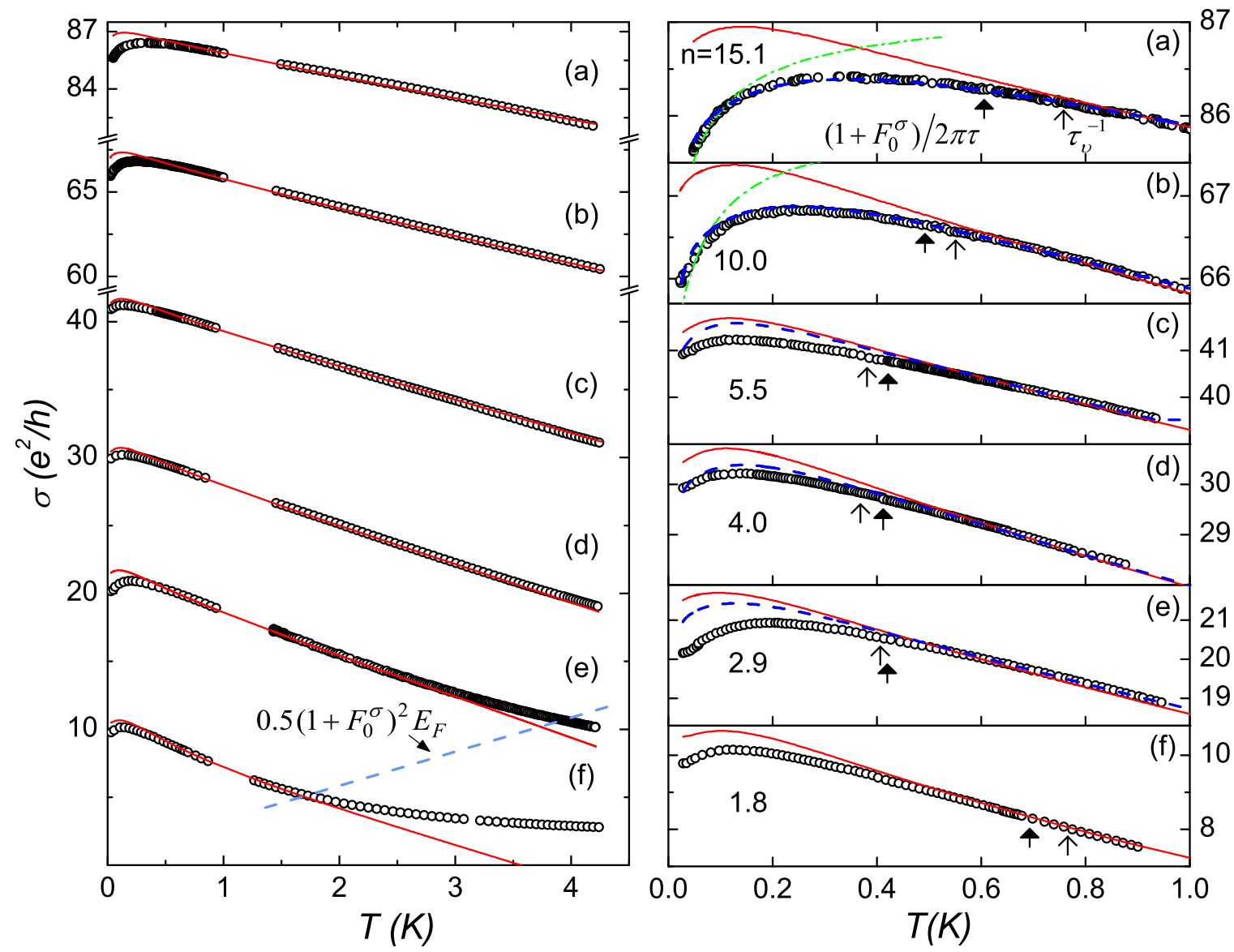

FIG. 2: (color online) Temperature dependences of the conductivity $\sigma\left(T, B_{\|}=0\right)$ for sample Si614 at various electron densities $n=15.1,10.0,5.5,4.0,2.9,1.8$, in units of $10^{11} \mathrm{~cm}^{-2}$, from top to bottom. Circles show experimental data, red curves - the theoretical dependences Eq. (5) calculated with $\Delta_{V}=0.4 \mathrm{~K}$. On the left panel, the dashed blue curve corresponds to $T=0.5\left(1+F_{0}^{\sigma}\right)^{2} E_{F}$, the applicability of the ZNA theory is violated at a higher $T$. The right panel shows the same data set within a narrower temperature interval, the thin arrows correspond to $T=\tau_{v}^{-1}$ and the thick arrows - to the temperature of the crossover between ballistic and diffusive regimes, $T^{*}=\left(1+F_{0}^{\sigma}\right) / 2 \pi \tau$. The dash-dotted green curves on the right panel were calculated with three triplet components in Eq. (1) (the valleys are completely intermixed), the blue dashed curves $\sigma(T, B)=\sigma_{D}+\delta \sigma_{C}+N_{\text {triplet }}\left(T \tau_{v}\right) \times \delta \sigma_{T}(T)$ with $N_{\text {triplet }}$ continuously varying between 3 (for $T \ll \tau_{v}^{-1}, \Delta_{V}$ ) and 15 (for $T \gg \tau_{v}^{-1}, \Delta_{V}$ ). 

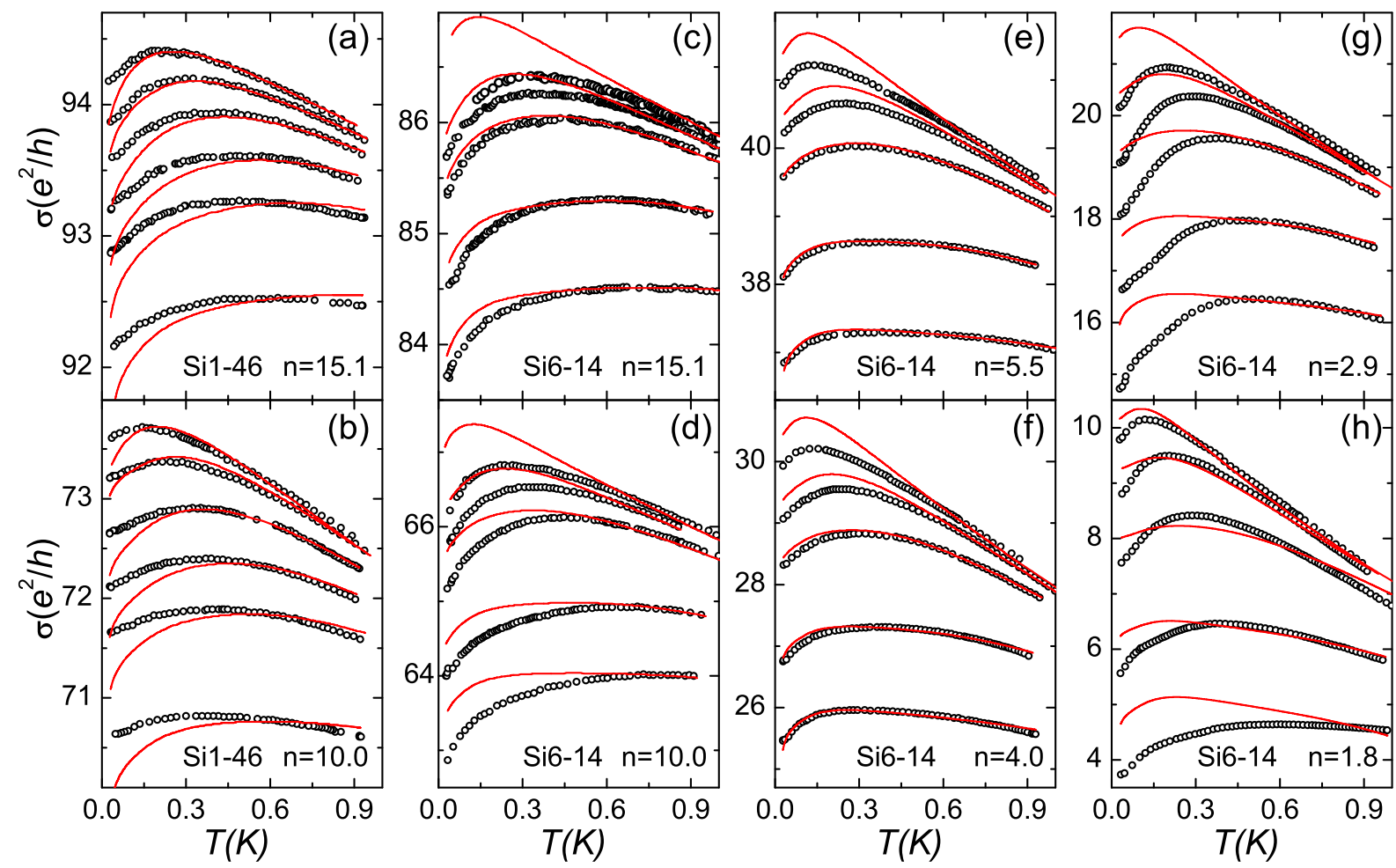

FIG. 3: (color online.) Temperature dependences of the conductivity for samples Si1-46 [(a) and (b)] and Si6-14 [(c) $\div(\mathrm{h})]$ in different in-plane magnetic fields (from top to bottom, $B_{\|}=$ $0,0.6,1,1.5,2,3 \mathrm{~T}$ in panels (a) and (b), $B_{\|}=0,0.6,1,2,3 \mathrm{~T}$ in panels (c) $\left.\div(\mathrm{h})\right)$. Experimental data are shown as circles, the solid curves show the theoretical dependences calculated for sample Si6-14 with $\Delta_{V}=0.4 \mathrm{~K}$ and for sample Si1-46 with $\Delta_{V}=0.7 \mathrm{~K}$. The $F_{0}^{\sigma}$ value is the only fitting parameter in comparison with the theory $\underline{26}^{26}$, the corresponding values of $F_{0}^{\sigma}$ are shown in Fig. 5 . The values of $n$ are shown in units of $10^{11} \mathrm{~cm}^{-2}$. 


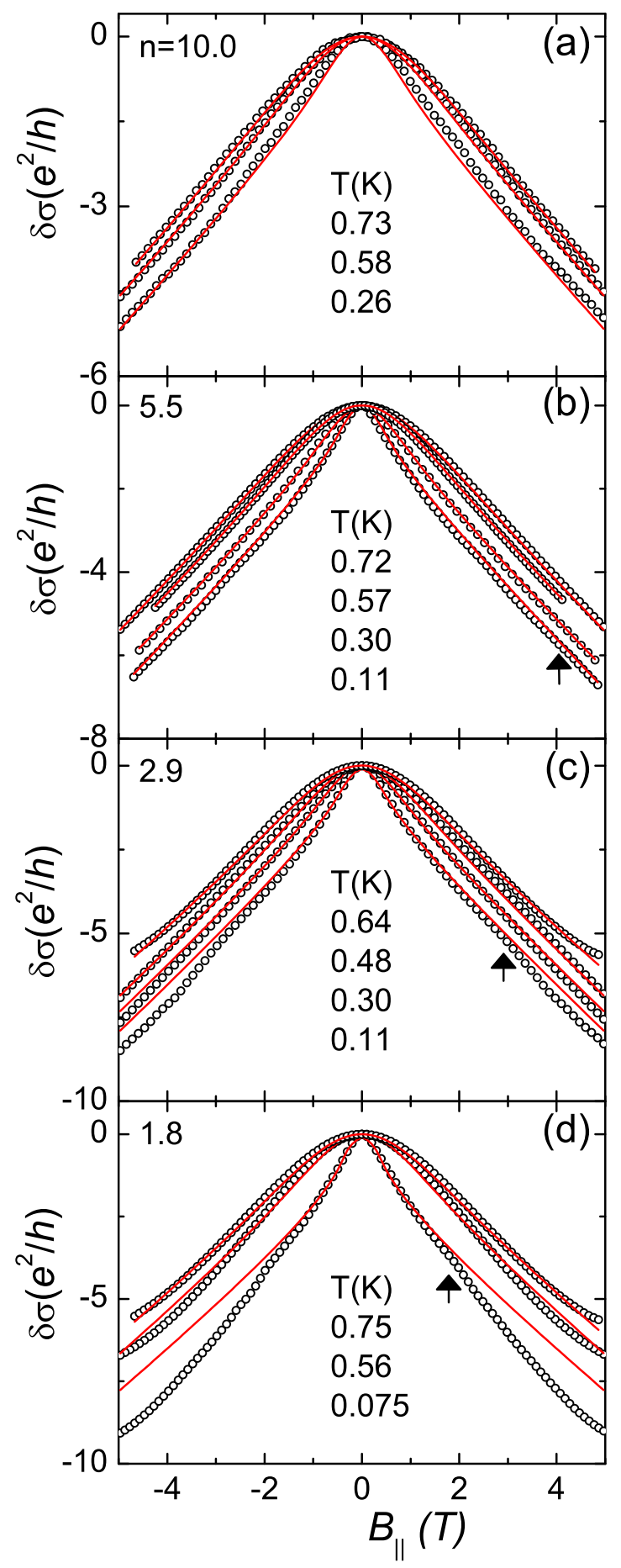

FIG. 4: (color online.) Magnetoconductance for sample Si6-14 at different electron densities and temperatures. Experimental data are shown by dots, the theoretical dependences calculated according to Eqs. $(5,6)$ - by solid curves. The $F_{0}^{\sigma}$ value is the only fitting parameter in comparison with the theory ${ }^{26}$, the corresponding values of $F_{0}^{\sigma}$ are shown in Fig. 5. Arrows indicate the fields corresponding to the condition $g \mu_{B} B_{\|} / 2 E_{F}=0.1$. The values of $n$ are shown in units of $10^{11} \mathrm{~cm}^{-2}$. 


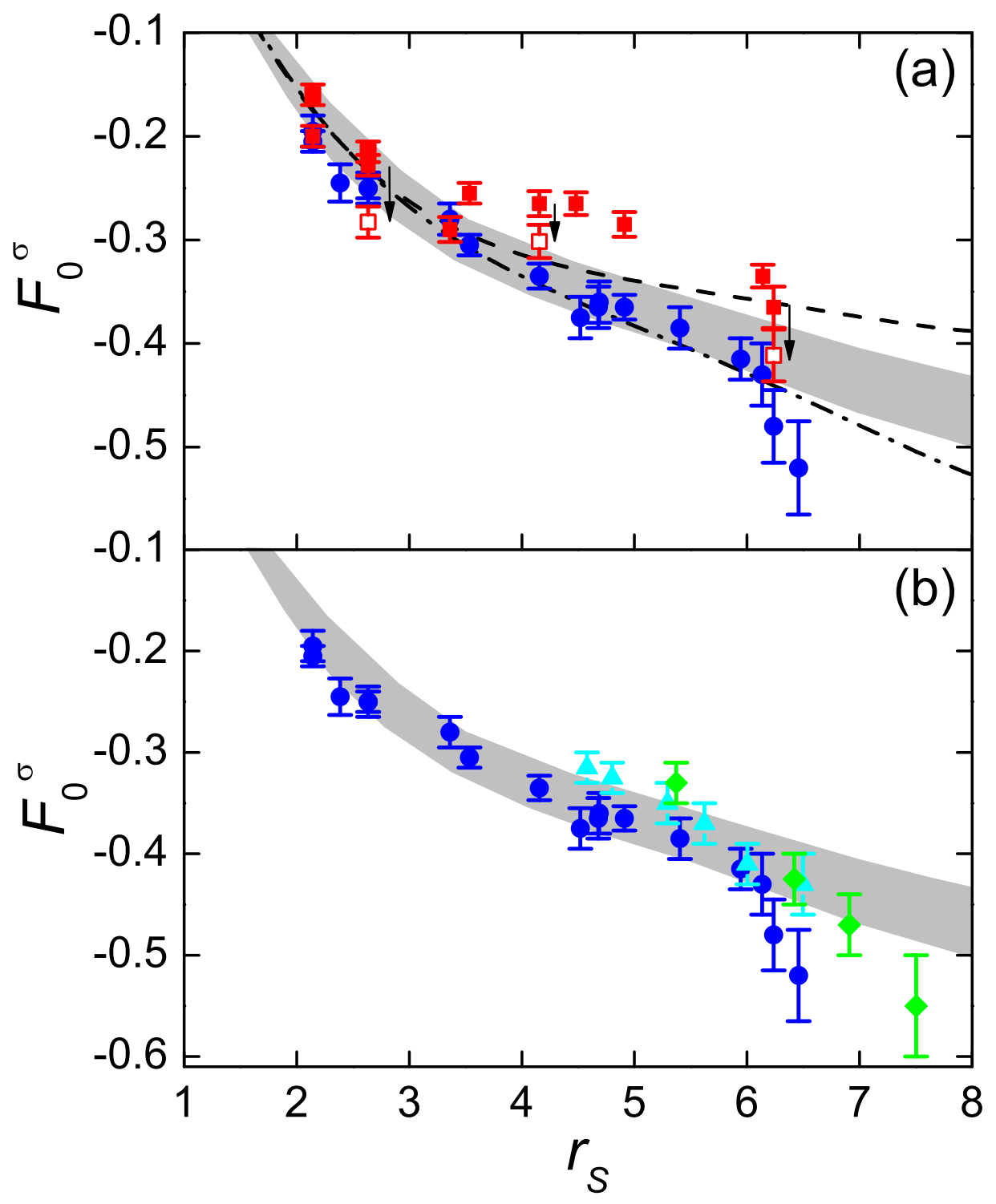

FIG. 5: (color online.) (a) The $F_{0}^{\sigma}$ values obtained from fitting the $\sigma(T)$ and $\sigma\left(B_{\|}\right)$dependences with the theory ${ }^{26}$ (blue and red symbols, respectively). Open red squares show the shift of several $F_{0}^{\sigma}$ values extracted from $\sigma\left(B_{\|}\right)$if one takes into account the $g\left(B_{\|}\right)$dependence and suppression of the WL corrections by $B_{\|}$(see the text). The dashed curve corresponds to $F_{0}^{\sigma}\left(r_{s}\right)$ extracted from the $\mathrm{SdH}$ data ${ }^{25}$ using the LK theory, the dash-dotted curve - to the empirical approach used in Ref. 25. The shaded regions in panels (a) and (b) show the $F_{0}^{\sigma}\left(r_{s}\right)$ dependence (with the experimental uncertainty) obtained from fitting our $\mathrm{SdH}$ data ${ }^{25}$ with the theory $\underline{47}$. (b) Comparison of $F_{0}^{\sigma}$ values calculated from $\sigma(T, B=0)$ using the same fitting procedure (see the text): $\bullet$ - present work, $\diamond$ and $\boldsymbol{\Delta}$ - data recalculated from Refs. 36 and $\underline{38}$, respectively. 